\title{
Mindful Yoga for women with metastatic breast cancer: design of a randomized controlled trial
}

\author{
James W. Carson ${ }^{1 *}$, Kimberly M. Carson ${ }^{1}$, Maren K. Olsen ${ }^{2}$, Linda Sanders ${ }^{3}$ and Laura S. Porter ${ }^{4}$
}

\begin{abstract}
Background: Women with metastatic breast cancer (MBC) have average life expectancies of about 2 years, and report high levels of disease-related symptoms including pain, fatigue, sleep disturbance, psychological distress, and functional impairment. There is growing recognition of the limitations of medical approaches to managing such symptoms. Yoga is a mind-body discipline that has demonstrated a positive impact on psychological and functional health in early stage breast cancer patients and survivors, but has not been rigorously studied in advanced cancer samples.

Methods: This randomized controlled trial examines the feasibility and initial efficacy of a Mindful Yoga program, compared with a social support condition that controls for attention, on measures of disease-related symptoms such as pain and fatigue. The study will be completed by December 2017. Sixty-five women with MBC age $\geq 18$ are being identified and randomized with a 2:1 allocation to Mindful Yoga or a support group control intervention. The 120-min intervention sessions take place weekly for 8 weeks. The study is conducted at an urban tertiary care academic medical center located in Durham, North Carolina. The primary feasibility outcome is attendance at intervention sessions. Efficacy outcomes include pain, fatigue, sleep quality, psychological distress, mindfulness and functional capacity at post-intervention, 3-month follow-up, and 6-month follow-up.

Discussion: In this article, we present the challenges of designing a randomized controlled trial with long-term follow-up among women with MBC. These challenges include ensuring adequate recruitment including of minorities, limiting and controlling for selection bias, tailoring of the yoga intervention to address special needs, and maximizing adherence and retention. This project will provide important information regarding yoga as an intervention for women with advanced cancer, including preliminary data on the psychological and functional effects of yoga for MBC patients. This investigation will also establish rigorous methods for future research into yoga as an intervention for this population.
\end{abstract}

Trial registration: ClinicalTrials.gov identifer: NCT01927081, registered August 16, 2013

Keywords: Breast cancer, Cancer pain, Yoga, Mindfulness, Research design

\footnotetext{
*Correspondence: carsonja@ohsu.edu

'Department of Anesthesiology \& Perioperative Medicine, Oregon Health \&

Science University, 3181 SW Sam Jackson Park Rd., Portland, OR 97239, USA

Full list of author information is available at the end of the article
}

(c) The Author(s). 2017 Open Access This article is distributed under the terms of the Creative Commons Attribution 4.0 International License (http://creativecommons.org/licenses/by/4.0/), which permits unrestricted use, distribution, and reproduction in any medium, provided you give appropriate credit to the original author(s) and the source, provide a link to the Creative Commons license, and indicate if changes were made. The Creative Commons Public Domain Dedication waiver (http://creativecommons.org/publicdomain/zero/1.0/) applies to the data made available in this article, unless otherwise stated. 


\section{Background}

Although metastatic breast cancer $(\mathrm{MBC})$ is a terminal illness, the development of more effective and better tolerated therapies has led to improved prognosis, with average life expectancies of 2 years or more. Nonetheless, women with MBC continue to report poor quality of life and high levels of pain and other disease-related symptoms including fatigue, sleep disturbance, psychological distress, and functional impairment [1]. Coping with these symptoms in the context of a life-limiting disease is very challenging $[2,3]$. There is growing recognition of the limitations of medical approaches to managing cancer pain and related symptoms, and heightened interest in the role that self-management interventions can play in cancer symptom control [4]. Considering the challenging course of MBC along with the high prevalence of pain and associated symptoms and impairments, it is critical to develop effective interventions to enhance the quality of the remaining years of these women's lives.

Recently there has been a growing interest in incorporating mind/body practices such as yoga and tai chi to help cancer patients cope with disease-related symptoms $[5,6]$. The Office of Cancer Complementary and Alternative Medicine has prioritized studies of yoga and other mind/body therapies, as has the National Center for Complementary and Integrative Health $[7,8]$. Yoga has been practiced in India for its proposed physical and mental benefits for thousands of years [9]. Yoga (which means 'union' in Sanskrit) aims at uniting mind and body through a variety of strategies such as practicing poses that promote strength, balance and flexibility [10-12]; breathing techniques that have soothing and energizing effects $[13,14]$; and meditation to develop mental calm and emotional clarity [15].

Over the last several decades yoga interventions have been demonstrated to positively impact a variety of medical conditions, including hypertension $[16,17]$, asthma [18], multiple sclerosis [19], tuberculosis [20], back pain $[21,22]$, fibromyalgia [23, 24], and neck pain [25]. Yoga has also shown promise for improving cancer-related symptoms such as fatigue and stress [26]. However prior studies in cancer patients have been largely limited to early stage breast cancer patients and survivors. Thus far, five systematic reviews of randomized controlled trials (RCTs) of yoga for cancer have been published [27-31]. Three reviews included meta-analyses [27, 28, 30], and one of the remaining two focused exclusively on fatigue outcomes [31]. The number of RCTs included across the reviews ranged from 10 to 18 , with sample sizes ranging from 18 to 164. Although the research quality across these studies varied greatly, on average the quality was relatively good (e.g., median quality $=67 \%$, range 22-89\% [27]). Most of the reviewed yoga interventions primarily emphasized physical poses, supplemented by some combination of breathing techniques, and relaxation or meditation practices. Approximately 50\% of RCTs compared yoga with wait-list control conditions, and most of the remaining studies employed supportive care control conditions [29].

The consensus conclusion across the reviews was that there is moderate to good evidence that, at least in the short-term, yoga has a positive impact on psychological health in early stage breast cancer patients and survivors. Effect sizes for psychosocial outcomes (e.g., emotional distress, anxiety, depression, global quality of life) were moderate to large, whereas for functional well-being effect sizes were small. Fatigue improved in approximately 50\% of studies where assessed. Only one RCT-which was conducted by the authors of this paper-assessed pain, which improved in that study [32]. None of the studies reported serious adverse events. Each of the available systematic reviews concluded that larger, more rigorous trials are needed and that these trials should include methodological improvements such as better control conditions, more physiological measures, longer term follow-up, and a wider range of cancer populations, including those with advanced cancer. A notable gap in the yoga for breast cancer literature is the absence of RCTs among individuals with advanced cancer. Recently, we conducted a pilot study to test a novel 8-week Mindful Yoga intervention designed to address the cancer-related pain, fatigue and emotional distress experienced by women with MBC. This intervention (previously published as 'Yoga of Awareness') showed promising results in terms of effects on these symptoms, and feasibility [33]. However, the MBC pilot study had major limitations including the lack of a control group, very small sample size $(N=13)$, and failure to address mediators that might explain the treatment effects.

In this paper, we present the design and detailed protocol of the first-known RCT of a yoga intervention for patients with advanced cancer. This single-blinded, randomized, attention-controlled, clinical trial for women with $\mathrm{MBC}$ has been funded by the National Center for Complementary and Integrative Health. Along with design considerations, we discuss the overall challenges of this trial as regards recruitment strategies, tailoring of the yoga intervention, and maximizing adherence and retention. We describe methods for addressing the theoretical and logistical issues encountered in conducting such a trial. Upon completion of the study, the results will be reported in accordance with the Consolidation of Standards for Reporting Trials (CONSORT) guidelines [34].

\section{Methods/Design \\ Study Design}

This is a randomized, controlled, single-blind, clinical trial of yoga in the treatment of cancer-related pain and associated symptoms in 65 women with metastatic breast cancer. Given the absence of prior yoga RCTs for 
patients with advanced cancer, our aims encompass the feasibility and acceptability of the Mindful Yoga intervention in this population, as well as comparing the efficacy of this intervention with a social support comparison condition. Feasibility will be indicated by our ability to meet accrual goals, and by at least $70 \%$ of patients attending $\geq 4$ of 8 sessions and providing posttest assessments. Acceptability will be indicated by $\geq 80 \%$ of participants reporting satisfaction with the yoga intervention on a standardized measure of treatment effectiveness/satisfaction. Regarding efficacy, we hypothesize that the yoga intervention will lead to reductions in pain, fatigue, sleep disturbance, and psychological distress and increases in functional capacity compared to a social support intervention.

The social support comparison was chosen for several reasons. First, we sought to address literature reviewers' criticism of the frequent employment of treatment-asusual and wait-list control conditions in yoga for cancer RCTs [27-31]. Such comparisons fail to control for any factor other than the general passage of time. Second, based on our prior research and reviewer's recommendations, we determined that the most important factors to control for were attention and time within a professionallyguided group intervention context. We ultimately decided on a social support comparison condition because previous studies indicate this type of intervention is viewed as highly credible by patients, and attendance at social support groups is comparable to that of other intervention groups [35-37]. Along with controlling for attention and time, this condition controls for nonspecific treatment effects such as general social support. The "single-blind" study design was chosen because it was not feasible to conceal assignment to the yoga intervention from participants and the intervention instructors. However, all study investigators and study staff involved in data collection will be masked to treatment condition, and statisticians will remain masked until the database is deemed final and ready for statistical analysis. As this is a low-risk behavioral trial, we do not foresee any circumstance under which blinded staff members will need to be unblinded during the trial.

Outcome assessments include intervention attendance rates, self-report measures of intervention satisfaction, pain, fatigue, sleep quality, psychological distress, mindfulness, a 6 min walk test measuring functional capacity, and treatment expectations. Assessments will be completed at baseline, post-treatment, and 3-month and 6-month follow-up. In addition, during the intervention period, participants will complete brief daily diaries on alternate weeks (4 weeks total) assessing symptoms (pain, fatigue, positive mood, negative mood, pain catastrophizing, pain acceptance) and yoga practice. An innovative feature of this study is that we will utilize these daily measures to conduct a rigorous test of two key psychological mediators that may explain the mechanisms of treatment effects. Pain theory and research point to two variables-pain catastrophizing (the tendency to focus on and exaggerate the threat value of painful stimuli and negatively evaluate one's own ability to deal with pain) and pain acceptance (the willingness to experience pain and yet remain engaged in meaningful life activities despite pain) - that are likely to be particularly important in explaining the effects of the yoga intervention on pain and pain-related outcomes $[38,39]$. Understanding the underlying mechanisms of the yoga intervention's potential effects could have far-reaching implications for the design and evaluation of future yoga interventions for conditions entailing persistent pain.

The setting of the study is an urban tertiary care academic medical center located in Durham, North Carolina (Duke Cancer Institute). This study has been reviewed and approved by the Duke Medical Center Institutional Review Board (IRB). The study will adhere to the CONSORT guidelines for clinical trials [34]. The study flow is presented in Fig. 1.

\section{Study population}

Participants in this study are women with age $\geq 18$ who are receiving treatment for metastatic breast cancer (Stage IV breast cancer or recurrent metastatic breast cancer). Participants must have a life expectancy $\geq$ 9 months as estimated by their treating oncologist; speak and read English; and be able to understand study procedures and comply with them for the entire length of the study period. Individuals will be excluded if they have: (a) cognitive impairment as assessed by the 6-item MiniMental Status Exam; (b) Eastern Cooperative Oncology Group (ECOG) rating of $\geq 3$ or Karnofsky Performance Status (KPS) $<60$ as rated by the oncology provider; (c) too sick to participate, as determined by the treating oncologist; (d) received treatment for serious psychiatric illness (e.g., schizophrenia, severe depression) in the past 6 months; (e) current engagement in yoga practice $\geq 1$ day per week; (f) resting systolic blood pressure $>180 \mathrm{~mm}$ and/or diastolic blood pressure $>100 \mathrm{~mm}$, or resting heart rate $>100$ beats per minute at the baseline assessment; $(\mathrm{g})$ unable or unwilling to give written informed consent.

During the trial, all participants will continue to receive the usual care provided by their health care providers. Medications and treatments received during the study are documented and examined later in data analyses, but not restricted.

\section{Recruitment strategies}

The percentage of MBC patients that accept entry when approached about behavioral intervention studies varies widely in the literature, from $27 \%$ [40] to $84 \%$ [41]. Our recruitment strategies focus on enrolling interested, 


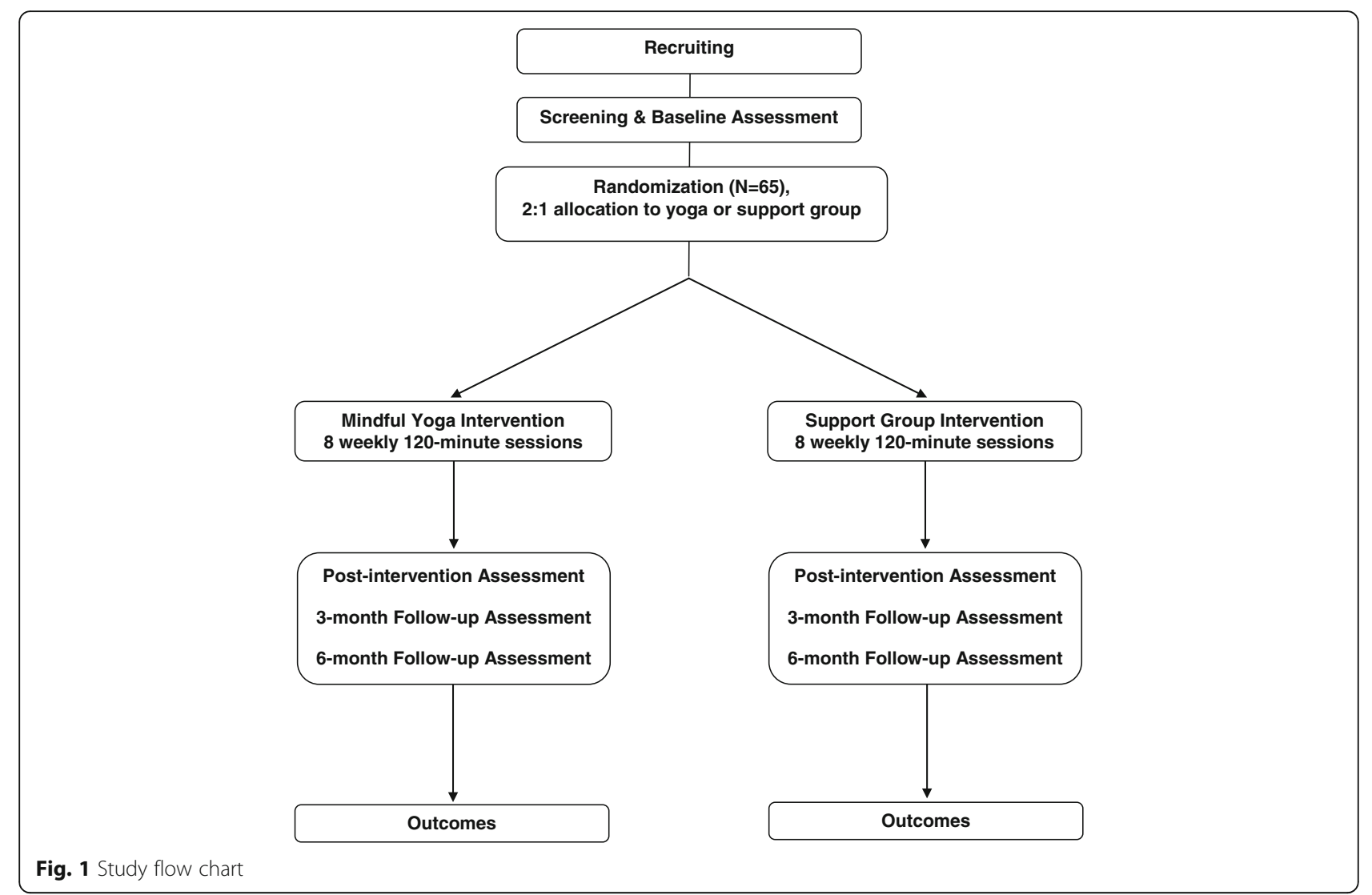

completely informed participants, and we have projected a modest $25 \%$ acceptance rate for the present study. To address the significant gaps in research participation among minorities that limit the generalizability of findings, especially among African Americans (38\% of the population in Durham County, North Carolina [42]), we are employing a variety of strategies to optimize minority participant involvement in the investigation.

Initial contact with potential participants is initiated by each patient's health care provider, either verbally or by an IRB-approved introductory letter and pamphlet. Given the rapidly expanding interest in yoga among breast cancer patients [43], the introductory materials describe the yoga intervention as a "self-management discussion group + gentle exercise", and the social support intervention as a "self-management discussion group", with the aim of minimizing selection bias related to patients' intervention preferences. This introduction includes graphics that are inclusive of various minority groups. Consistent with recommendations on the recruitment of African-American participants for clinical and prevention trials, particularly in oncology, we provide African-American (and all) potential participants with an opportunity to review the information about the study without any direct request to participate. Individuals who do not refuse further contact are called by a clinical research coordinator who has received training in how to address the social and cultural barriers to engaging African American cancer survivors in behavioral treatment studies. The study coordinator provides a brief scripted explanation of the study, answers any questions, and verifies eligibility. If the patient is eligible and chooses to participate, arrangements are made to obtain written consent and to administer the baseline assessment. As an added incentive, each participant is paid $\$ 190$ for full participation in the study to offset costs participants might incur by participating (e.g., babysitters during sessions) and as an honorarium for their time.

\section{Adherence and retention strategies}

Adherence and retention are crucial determinants of the quality and interpretability of clinical trials. Obstacles to treatment attendance when intervening with patients with advanced disease include a variety of illness-related issues, such as unpredictable worsening of symptoms, limited energy, and alternate patient priorities given shortened life expectancies. Strategies we have implemented to boost attendance are among the best practices used in many high quality behavioral intervention trials. These strategies include motivational interviewing during the recruitment phase, weekly reminder emails during the intervention phase, prompt calls from interventionists to participants 
who missed a class, and make up sessions. Adequate adherence in this trial has been defined as $70 \%$ of the participants attending full sessions (120 min each) for at least 4 of the 8 treatment sessions. This is a revision from the initial definition of adequate adherence, which specified 70\% attendance of at least 7 intervention sessions. We believe that the revision represents a more reasonable expectation given the severity of illness in this patient population. In reviewing data from three previous studies of the 8session Mindful Yoga intervention, it was apparent that the goal of $70 \%$ of participants attending at least 7 sessions was likely overly ambitious [32, 33, 44]. This was achieved in only one of the three previous studies, with a population of much healthier women who were post-treatment for early stage breast cancer [32].

Attrition is a recognized challenge in studying the $\mathrm{MBC}$ population, given the progressive nature of the disease. To minimize missing data, we developed a retention plan to ensure minimal dropout due to reasons other than death or severe disease progression. This plan has featured minimization of participant burden, provision of participant support through the intervention protocols, solicitation of information about participant difficulties and prompt response to concerns raised, and consistent efforts to optimize the participants' experience of study participation. Continuous quality monitoring of retention key performance indicators has allowed us to rapidly identify and respond to problems.

\section{Sample size}

Our original sample size of 60 patients (40 intervention and 20 control) was based mainly on the primary aim of intervention feasibility and acceptability. While we have increased the targeted enrollment to approximately 65 patients, statistical power to detect significant intervention differences remains limited. Nonetheless, our sample size will be sufficient to conduct longitudinal analyses of the outcome variables to inform the design of a larger-scale, multisite trial.

\section{Randomization}

Participants are randomized with a 2:1 allocation to: (a) Mindful Yoga, or (b) a support group control intervention. The study statisticians (M.O. \& L.S.) generated the randomization scheme prior to the start of recruitment, which is kept in a study database which is not accessible by blinded study personnel. The study coordinator who executes the randomization schedule does not have access to the data and is not involved in the outcome assessments.

Due to the group nature of the interventions, enrollment has proceeded in cohorts. Randomization has been stratified within each cohort using variable block sizes of 3 and 6. The study coordinator informs each participant of their assigned treatment condition following the baseline assessment and $\leq 14$ days prior to the start of the interventions.

We planned to conduct cohorts of 20-25 participants, so that each intervention group (two yoga groups and one social support group per cohort) would have between 5 and 10 group members. After the first two cohorts, we found that class size was an important factor in the yoga classes, which functioned best with at least five patients in attendance, but that class size did not appear to impact the functionality of the social support classes. Given that attendance at each class is usually less than $100 \%$, it is necessary to assign at least seven patients to each yoga class to meet the expectation of usually having at least five patients in attendance. This necessitates enrolling at least 20 patients per cohort to fill two yoga classes and one social support class.

\section{Intervention}

Both interventions consist of eight group sessions conducted weekly in 120-min sessions (Fig. 1).

\section{Mindful Yoga Program}

This intervention consists of eight 120-min weekly group sessions led by a certified yoga instructor who has extensive experience in teaching yoga techniques to medical patients. The Mindful Yoga Program is based in part on the Kripalu school of yoga, which is widely taught in the U.S. [45, 46]. Mindful Yoga can be distinguished from many current yoga styles in that it places strong emphasis on developing mindfulness via substantial meditation practice, breathing exercises, study of yoga philosophy, practitioner meetings, and informal application of mindful awareness in daily life [26]. Mindfulness entails strategies for developing greater moment-tomoment presence of mind, along with acceptance of and willingness to learn from stressful experiences, so as to begin to recognize clearly what choices contribute to more wellbeing versus suffering [39, 47, 48]. Accordingly, during posture exercises Mindful Yoga intensively highlights the inner dimensions of practice-such as nonreactive monitoring of sensory, mental and emotional fluctuations-along with proper alignment and breathing techniques. The yoga instructor follows a detailed intervention manual (Carson JW, Carson KM: Mindful Yoga Professional Training Manual, unpublished). Each session includes gentle postures (approx. $40 \mathrm{~min})$, breathing techniques (10 $\mathrm{min})$, meditation (25 min), presentations on the application of yogic principles to optimal coping (e.g., mind/body stress reactions; $20 \mathrm{~min}$ ), and group discussions (e.g., experiences during meditation; $25 \mathrm{~min}$ ). The yoga poses included in this protocol carry no more risk of injury than everyday activities such as climbing stairs. They have been specifically selected and tailored to meet the needs of women 
with $\mathrm{MBC}$, and the instructor emphasizes gentle performance. Patients are supplied with yoga mats, eye pillows, and bolsters for doing poses. Participants are encouraged to practice yoga techniques at home, 15-30 min per day, 5-6 days per week, guided by professionally produced video recordings. Participants also receive brief session summary handouts each week, which include instructions for the informal application of yoga practice to daily life (e.g., in-the-moment acceptance of pain).

\section{Social support comparison condition}

This intervention consists of eight 120-min weekly group sessions led by a licensed clinical social worker experienced in leading groups and in working with patients with advanced cancer. The scheduling of the sessions is identical to that for the yoga intervention. The social support intervention is modeled after the protocol utilized by Breitbart and colleagues, and the therapist follows a detailed treatment manual [35]. The sessions focus on discussion of issues and themes that emerge for patients coping with $\mathrm{MBC}$, including the following: coping with medical tests; communicating with healthcare providers; coping with family and friends; vocational issues; body image and physical functioning concerns; fears about future physical or psychological changes, recurrence, and mortality; and plans for the future. Utilizing a supportive approach, the therapist focuses on encouraging patients to share concerns related to the cancer diagnosis and treatment, to describe their experiences and emotions related to these experiences, to voice problems that they have in coping with cancer, and to offer support and advice to other group members.

\section{Measures}

All participants complete assessments at baseline, posttreatment, and 3-month and 6-month follow-up (see Table 1). In addition, they complete daily diary measures during alternating weeks during the intervention period assessing symptoms and yoga practice (for those in the yoga condition).

\section{Attendance}

Attendance and time spent at each session are recorded by having participants sign in and out of each session,

Table 1 Sequence of trial measurements for primary and secondary outcomes

\begin{tabular}{|c|c|c|c|c|c|c|c|c|c|}
\hline & $\begin{array}{l}\text { Pre- } \\
\text { Screening }\end{array}$ & $\begin{array}{l}\text { Telephone } \\
\text { Screening }\end{array}$ & Baseline & Treatment & Mid-Treatment & Treatment & $\begin{array}{l}\text { Post- } \\
\text { Treatment }\end{array}$ & $\begin{array}{l}\text { 3-Month } \\
\text { Follow-up }\end{array}$ & $\begin{array}{l}\text { 6-Month } \\
\text { Follow-up }\end{array}$ \\
\hline VISIT, Time (weeks) & $(-16-0)$ & $(-13-0)$ & $1(-4-0)$ & $2-4(1-3)$ & $5(4)$ & $6-9(6-8)$ & $10(9)$ & $11(21)$ & $12(34)$ \\
\hline \multicolumn{10}{|l|}{ Primary outcome measures } \\
\hline $\begin{array}{l}\text { Attendance at intervention } \\
\text { sessions }\end{array}$ & & & & $x$ & & $x$ & & & \\
\hline \multicolumn{10}{|l|}{ Client Satisfaction Questionnaire-8 } \\
\hline \multicolumn{10}{|l|}{ Secondary outcome measures } \\
\hline Brief Pain Inventory-Short Form & & & $x$ & & & & $x$ & $x$ & $x$ \\
\hline Brief Fatigue Inventory & & & $x$ & & & & $x$ & $x$ & $x$ \\
\hline Pittsburg Sleep Quality Index & & & $x$ & & & & $x$ & $x$ & $x$ \\
\hline $\begin{array}{l}\text { Hospital Anxiety \& Depression } \\
\text { Scale }\end{array}$ & & & $x$ & & & & $x$ & $x$ & $x$ \\
\hline $\begin{array}{l}\text { Mindfulness questionnaire } \\
\text { (FFMQ-SF) }\end{array}$ & & & $x$ & & & & $x$ & $x$ & $x$ \\
\hline $\begin{array}{l}\text { Complementary/alternative } \\
\text { medicine use }\end{array}$ & & & $x$ & & & & $x$ & $x$ & $x$ \\
\hline 6-min Walk Test & & & $x$ & & & & $x$ & $x$ & $x$ \\
\hline Medical record review & $x$ & & $x$ & & & & $x$ & $x$ & $x$ \\
\hline ECOG or KPS & $x$ & & & & & & & & \\
\hline Demographics & & & $x$ & & & & & & \\
\hline Daily diary items & & & & $x$ & & $x$ & & & \\
\hline Medication log & & & $x$ & & $x$ & & $x$ & $x$ & $x$ \\
\hline Health questionnaire & & & $x$ & & & & & & \\
\hline Treatment expectations & & & $x$ & & $x$ & & & & \\
\hline Adverse events & & & $x$ & $x$ & $x$ & $x$ & $x$ & $x$ & $x$ \\
\hline
\end{tabular}


including the precise time at which they enter and leave. The interventionist verifies the times recorded.

\section{Satisfaction with intervention}

This is assessed with the 8-item Client Satisfaction Questionnaire-8, a briefer version of the larger Client Satisfaction Questionnaire [49]. This measure is frequently used to assess participants' satisfaction with, and impression of the effectiveness of, the services they received. Items are rated on a four-point scale. This measure has good reliability (alpha coefficient $=.93$ ). It is administered at the post-treatment assessment only.

\section{Pain}

Pain is assessed with the Brief Pain Inventory-Short Form, a 9-item self-report measure that assesses worst, least, and average levels of pain and the degree which pain interferes in activities, mood, relationships, sleep, and enjoyment of life. This measure is widely used with cancer patients, has evidence of reliability and validity, and is considered the preferred method of assessing pain endpoints [50, 51].

\section{Fatigue}

The Brief Fatigue Inventory assesses self-reported levels of current, worst, and usual fatigue, and interference due to fatigue. The measure consists of 9 items on which respondents choose a number from 0 to 10 to rate each item on the scale. Factor analysis has shown that $75 \%$ of the variance on the Brief Fatigue Inventory can be explained by a single factor suggesting that it measures a single construct. This measure has also shown good evidence of concurrent and discriminant validity as well as excellent internal consistency (alpha $=$.96.) [52].

\section{Sleep quality}

Sleep quality is assessed by the Pittsburgh Sleep Quality Index, a 19-item self-report measure that produces a total sleep quality score and seven sleep component scores [53]. Higher scores indicate poorer sleep quality. The scale has been widely used with breast cancer patients with internal consistency for the total scale reported as .78 [54].

\section{Psychological distress}

The Hospital Anxiety and Depression Scale (HADS) is used to assess depression and anxiety symptoms. The HADS is a 14-item, 2-domain (depression and anxiety) scale with evidence of reliability, validity, and responsiveness among cancer patients $[55,56]$. Domain scores $\geq 8$ indicate either likely depression or anxiety.

\section{Mindfulness}

The Five Facet Mindfulness Questionnaire-Short Form (FFMQ-SF) assesses different aspects of mindfulness [57]. The FFMQ-SF is a 24-item short form of the Five
Facet Mindfulness Questionnaire, a comprehensive measure for assessing mindfulness [58]. The FFMQ-SF has demonstrated reliability and validity as well as sensitivity to change in samples of adults with anxiety and depression as well as fibromyalgia [57].

\section{Functional capacity}

Patients' functional capacity is assessed by the 6-min Walk Test administered in a measured corridor according to American Thoracic Society (ATS) guidelines [59]. Patients are instructed to walk at their fastest pace and to cover the longest possible distance over $6 \mathrm{~min}$. This test has been shown to be a reliable and sensitive measure of functional capacity in deconditioned clinical populations and a sensitive test to assess therapeutic response. The test has been successfully used in several prior studies of cancer patients, including patients with advanced disease, with no adverse events [60]. This test has been shown to be a strong predictor of all-cause mortality in lung cancer patients [61].

\section{Daily pain, fatigue, positive mood, negative mood, pain catastrophizing, and pain acceptance}

Daily variation in pain, fatigue, positive mood, and negative mood is each assessed with a single 0-9 scale item. Similar numeric scales are extensively used in clinical and research settings to measure subjective phenomena and have been found to be valid and reliable [62]. Pain catastrophizing is measured using a well-validated two item measure rated on a 0-6 scale [63]. Pain acceptance is measured using two items from the Chronic Pain Acceptance Questionnaire (CPAQ), the only well-validated measure of pain acceptance [64]. The two CPAQ items were chosen based on their high loadings on each of the CPAQ's two subscales, willingness to experience pain and engaging in activities despite pain [65].

\section{Treatment expectations}

We will examine treatment expectations using the 9-item Outcome Expectations for Exercise Scale, in which participants are asked to rate their agreement with potential benefits of physical activity measured on a 5-point Likerttype scale, with 1 indicating no expectations and 5 the highest expectations for exercise. Validity and reliability has been previously established for this measure [66].

\section{Safety}

Study participants are monitored weekly during the intervention period for the occurrence of adverse events, and at each assessment (baseline, post-treatment, 3 months follow-up, and 6 months follow-up). An adverse event is defined as any unfavorable and unintended diagnosis, symptom, sign (including an abnormal laboratory finding), syndrome or disease which either occurs during the study, 
having been absent at baseline, or if present at baseline, appears to worsen. Adverse events are recorded regardless of their relationship to the study intervention. Solicited adverse events include psychological distress (HADS score $\geq 15$ ), and heart rate, blood pressure, and oxygen saturation collected during the 6-min Walk Test. In addition, information about muscle soreness is collected at each session of the yoga intervention.

\section{Statistical analysis}

The primary outcome related to feasibility is attendance, which is measured weekly at each intervention session. Adequate feasibility will be indicated by at least $70 \%$ of patients attending $\geq 4$ of 8 sessions. The primary outcome related to acceptability is quantified by the Client Satisfaction Questionnaire-8 as assessed at the post-intervention evaluation. Adequate acceptability of the yoga intervention will be indicated by $\geq 80 \%$ of participants reporting a mean satisfaction score $\geq 3$. Secondary outcomes are measurements of change at the post-treatment, 3-month follow-up, and 6month follow-up evaluations in pain, fatigue, sleep quality, psychological distress, mindfulness and functional capacity. These will be analyzed both as individual time points and in longitudinal analyses. Treatment expectations-including potential pre-randomization selection biases regarding the yoga intervention-will be controlled in analyses with the data from the measure assessing outcomes expectations [66]. We will apply the intent-to-treat assumption for all analyses.

We will also explore within-person associations between yoga practice, outcomes (pain, fatigue, mood) and mediators (pain catastrophizing, pain acceptance) among participants in the yoga condition. Multilevel random effects models will be used to evaluate these associations, with individuals' mean levels of yoga practice as a control variable, and practice rates person-centered to control for potentially spurious within-person associations [67]. Multilevel modeling is an advanced methodology for integrating data from multiple levels of sampling, such as this study's two levels (withinpersons and between-persons). Multilevel models are particularly advantageous in analyzing data sets with many repeated measures, such as daily diary records $[68,69]$. By preserving the rich detail in each individual's full data set, multilevel models allow for a sensitive independent determination of day-to-day interrelated happenings for each patient, as well as aggregation of individual estimates for reliable results for the average patient. Multilevel models also enable strict control for potential confounds, such as serial autocorrelation in measurements.

\section{Discussion}

In this report we have summarized the challenges of designing a randomized controlled trial of a yoga intervention for metastatic breast cancer patients with long-term follow-up. The challenges we faced in this design included ensuring adequate recruitment including of minorities, limiting and controlling for selection bias, tailoring of the yoga intervention, and maximizing adherence and retention. This project will provide important information regarding the feasibility and acceptability of yoga as an intervention for women with advanced cancer, and valuable preliminary data on the psychological and functional effects of yoga for MBC patients, including the first systematic evaluation of the psychological mechanisms by which a yoga intervention may produce pain relief. This investigation will also establish rigorous methods for future research for testing the mechanisms by which yoga may affect pain, fatigue, emotional distress, and functional capacity in this population.

\section{Abbreviations \\ CONSORT: Consolidation of Standards for Reporting Trials; CPAQ: Chronic Pain Acceptance Questionnaire; ECOG: Eastern Cooperative Oncology Group; FFMQ- SF: Five Facet Mindfulness Questionnaire-Short Form; HADS: Hospital Anxiety and Depression Scale; IRB: Institutional Review Board; KPS: Karnofsky Performance Status; MBC: Metastatic breast cancer; RCT: Randomized controlled trial \\ Acknowledgements \\ Dr Porter is supported by R01 AT007572 from NCCIH. The contents of this manuscript are solely the responsibility of the authors and do not necessarily represent the views of $\mathrm{NCCIH}$. The authors gratefully acknowledge the Data Safety Monitoring Board members: Diana Wilkie, PhD, RN, FAAN; Mary Jane Ott, NP, MN, RYT; Christopher Corcoran, PhD; and Beverly Moy, MD for their insightful suggestions and comments for the study protocol.}

\section{Funding}

This study is funded by the National Center for Complementary and Integrative Health (R01 AT007572).

Availability of data and materials

This trial is registered at ClinicalTrials.gov (NCT01927081) where current data and materials are available to the public

\section{Authors' contributions}

LP obtained funding for the study. LP, JC, KM, and MO participated in designing the randomized controlled trial. All authors have been involved in conducting the research. JC wrote the first draft of the manuscript. All authors participated in the revision of subsequent drafts, and all approved the final version of the manuscript.

\section{Competing interests}

The authors declare that there is no conflict of interest.

Consent for publication

Not applicable.

Ethics approval and consent to participate

This study has been reviewed and approved by the Duke Medical Center Institutional Review Board (IRB).

\section{Publisher's Note}

Springer Nature remains neutral with regard to jurisdictional claims in published maps and institutional affiliations.

\section{Author details}

${ }^{1}$ Department of Anesthesiology \& Perioperative Medicine, Oregon Health \& Science University, 3181 SW Sam Jackson Park Rd., Portland, OR 97239, USA

${ }^{2}$ Center for Health Services Research in Primary Care, Durham VA Medical Center, Durham, NC 27705, USA. ${ }^{3}$ Department of Medicine, Duke University Medical Center, Box 2628, Durham, NC 27710, USA. ${ }^{4}$ Department of Psychiatry and Behavioral Sciences, Duke University Medical Center, Box 90399, Durham, NC 27708, USA. 


\section{Received: 25 February 2017 Accepted: 8 March 2017}

Published online: 13 March 2017

\section{References}

1. Irvin WJ, Muss HB, Mayer DK. Symptom management in metastatic breast cancer. Oncologist. 2011;16(9):1203-14.

2. Cella D. Factors influencing quality of life in cancer patients: anemia and fatigue. Semin Oncol. 1998;25:43-6.

3. Cleeland CS, Chapman CR, Foley KM. Documenting barriers to cancer pain management. In: Current and Emerging Issues in Cancer Pain Management: Research and Practice. New York: Raven; 1993.

4. Gordon DB, Dahl JL, Miaskowski C, McCarberg B, Todd KH, Paice JA, Lipman AG, Bookbinder M, Sanders SH, Turk DC, et al. American pain society recommendations for improving the quality of acute and cancer pain management: American Pain Society Quality of Care Task Force. Arch Intern Med. 2005;165(14):1574-80.

5. Tai Chi Exercise Studied to Improve Quality of Life for Senior Cancer Survivors. Office of Cancer Complementary and Alternative Medicine. [https://cam.cancer.gov/cam_at_nci/annual_report/2010/tai_chi_survivors. htm]. Accessed 16 Oct 2016.

6. Yoga and Cancer. Office of Cancer Complementary and Alternative Medicine. [https://cam.cancer.gov/health_information/highlights/yoga_ cancer_highlight.htm]. Accessed 23 Sept 2016.

7. Mind and Body Research-Information for Researchers. National Center for Complementary and Integrative Health. [https://nccih.nih.gov/grants/ mindbody]. Accessed 23 Sept 2016

8. Research Priorities. Office of Cancer Complementary and Alternative Medicine. [https://cam.cancer.gov/about_us/about_occam.htm\#priorities]. Accessed 23 Sept 2016.

9. Telles S, Naveen KV. Yoga for rehabilitation: an overview. Indian J Med Sci. 1997:51(4):123-7.

10. Hart CEF, Tracy BL. Yoga as steadiness training: effects on motor variability in young adults. J Strength Conditioning Res. 2008;22(5):1659-69.

11. Krishnamurthy M, Telles S. Effects of Yoga and an Ayurveda preparation on gait, balance and mobility in older persons. Med Sci Monit. 2007;13(12):LE19-20.

12. Raub JA, Full N. Psychophysiologic effects of Hatha Yoga on musculoskeletal and cardiopulmonary function: a literature review. J Altern Complement Med. 2002;8(6):797-812.

13. Busch V, Magerl W, Kern U, Haas J, Hajak G, Eichhammer P. The effect of deep and slow breathing on pain perception, autonomic activity, and mood processing-an experimental study. Pain Med. 2012;13(2):215-28.

14. Dhruva A, Miaskowski C, Abrams D, Acree M, Cooper B, Goodman S, Hech FM. Yoga breathing for cancer chemotherapy-associated symptoms and quality of life: results of a pilot randomized controlled trial. J Altern Complement Med. 2012;18(5):473-9.

15. Holzel BK, Lazar SW, Gard T, Schuman-Olivier Z, Vago DR, Ott U. How does mindfulness meditation work? Proposing mechanisms of action from a conceptual and neural perspective. Perspect Psychological Sci. 2011;6(6): 537-59.

16. Murugesan R, Govindarajulu N, Bera TK. Effect of selected yogic practices on the management of hypertension. Indian J Physiol Pharmacol. 2000;44(2):207-10.

17. Sundar S, Agrawal SK, Singh VP, Bhattacharya SK, Udupa KN, Vaish SK. Role of yoga in management of essential hypertension. Acta Cardiol. 1984;39(3):203-8

18. Manocha R, Marks GB, Kenchington P, Peters D, Salome CM. Sahaja yoga in the management of moderate to severe asthma: a randomised controlled trial. Thorax. 2002:57(2):110-5.

19. Oken BS, Kishiyama S, Zajdel D, Bourdette D, Carlsen J, Haas M, Hugos C, Kraemer DF, Lawrence J, Mass M. Randomized controlled trial of yoga and exercise in multiple sclerosis. Neurology. 2004;62(11):2058-64.

20. Visweswaraiah NK, Telles S. Randomized trial of yoga as a complementary therapy for pulmonary tuberculosis. Respirology. 2004;9(1):96-101.

21. Sherman KJ, Cherkin DC, Erro J, Miglioretti DL, Deyo RA. Comparing yoga, exercise, and a self-care book for chronic low back pain: a randomized, controlled trial. Ann Intern Med. 2005;143(12):849-56.

22. Williams K, Abildso C, Steinberg L, Doyle E, Epstein B, Smith D, Hobbs G, Gross R, Kelley G, Cooper L. Evaluation of the effectiveness and efficacy of lyengar yoga therapy on chronic low back pain. Spine. 2009;34(19):2066-76.

23. Carson JW, Carson KM, Jones KD, Bennett RM, Wright CL, Mist SD. A pilot randomized controlled trial of the Yoga of Awareness program in the management of fibromyalgia. Pain. 2010;151(2):530-9.
24. Carson JW, Carson KM, Jones KD, Lancaster L, Mist SD. Mindful yoga pilot study shows modulation of abnormal pain processing in fibromyalgia patients. Int J Yoga Ther. 2016;26:93-100.

25. Cramer H, Lauche R, Hohmann C, Ludtke R, Haller H, Michalsen A, Langhorst J, Dobos G. Randomized-controlled trial comparing yoga and home-based exercise for chronic neck pain. Clin J Pain. 2013;29(3):216-23.

26. Carson JW, Carson KM. Yoga as supportive care for healing from cancer. Yoga Ther Today. 2015;11:18-22.

27. Buffart LM, van Uffelen JGZ, Riphagen II, Brug J, van Mechelen W, Brown WJ, Chinapaw MJM. Physical and psychosocial benefits of yoga in cancer patients and survivors, a systematic review and meta-analysis of randomized controlled trials. BMC Cancer. 2012;12:559.

28. Cramer H, Lange S, Klose P, Paul A, Dobos G. Yoga for breast cancer patients and survivors: a systematic review and meta-analysis. BMC Cancer. 2012;12:412

29. Harder $H$, Parlour $L$, Jenkins $V$. Randomised controlled trials of yoga interventions for women with breast cancer: a systematic literature review. Support Care Cancer. 2012;20(12):3055-64.

30. Lin KY, Hu YT, Chang KJ, Lin HF, Tsauo JY. Effects of yoga on psychological health, quality of life, and physical health of patients with cancer: a metaanalysis. Evidence-Based Complementary Alternative Med. 2011;2011:659876.

31. Sadja J, Mills PJ. Effects of yoga interventions on fatigue in cancer patients and survivors: a systematic review of randomized controlled trials. Explore. 2013;9(4):232-43.

32. Carson JW, Carson KM, Porter LS, Keefe FJ, Seewaldt VL. Yoga of Awareness program for menopausal symptoms in breast cancer survivors: Results from a randomized trial. Support Care Cancer. 2009;17:1301-9.

33. Carson JW, Carson KM, Porter LS, Keefe FJ, Shaw H, Miller JM. Yoga for women with metastatic breast cancer: results from a pilot study. J Pain Symptom Manage. 2007;33(3):331-41.

34. Hopewell S, Clarke M, Moher D, Wager E, Middleton P, Altman DG, Schulz KF, Group C. CONSORT for reporting randomized controlled trials in journal and conference abstracts: explanation and elaboration. PLoS Med. 2008;5(1):e20.

35. Breitbart W, Rosenfeld B, Gibson C, Pessin H, Poppito S, Nelson C, Tomarken A, Timm AK, Berg A, Jacobson C, et al. Meaning-centered group psychotherapy for patients with advanced cancer: a pilot randomized controlled trial. Psychooncology. 2010;19(1):21-8.

36. Helgeson VS, Cohen S, Schulz R, Yasko J. Education and peer discussion group interventions and adjustment to breast cancer. Arch Gen Psychiatry. 1999:56(4):340-7.

37. Keefe FJ, Caldwell DS, Baucom D, Salley A, Robinson E, Timmons K, Beaupre P, Weisberg J, Helms M. Spouse-assisted coping skills training in the management of osteoarthritic knee pain. Arthritis Care Res. 1996;9(4):279-91.

38. Keefe FJ, Rumble ME, Scipio CD, Giordano LA, Perri LM. Psychological aspects of persistent pain: current state of the science. J Pain. 2004:5(4):195-211.

39. McCracken LM, Carson JW, Eccleston C, Keefe FJ. Acceptance and change in the context of chronic pain. Pain. 2004;109(1-2):4-7.

40. Cunningham AJ, Edmonds CV, Jenkins GP, Pollack H, Lockwood GA, Warr D. A randomized controlled trial of the effects of group psychological therapy on survival in women with metastatic breast cancer. Psychooncology. 1998; 7(6):508-17.

41. Classen C, Butler LD, Koopman C, Miller E, DiMiceli S, Giese-Davis J, Fobair P, Carlson RW, Kraemer HC, Spiegel D. Supportive-expressive group therapy and distress in patients with metastatic breast cancer: a randomized clinical intervention trial. Arch Gen Psychiatry. 2001;58(5):494-501.

42. QuickFacts Durham County, North Carolina. United States Census Bureau. [http://www.census.gov/quickfacts/table/RHI225215/37063]. Accessed 14 Oct 2016.

43. Sharma M, Haider T, Knowlden AP. Yoga as an alternative and complementary treatment for cancer: a systematic review. J Altern Complement Med. 2013;19(11):870-5.

44. Carson JW, Carson KM, Jones KD, Mist SD, Bennett RM. Follow-up of yoga of awareness for fibromyalgia: results at 3 months and replication in the waitlist group. Clin J Pain. 2012:28(9):804-13.

45. About Kripalu Center for Yoga and Health. Yoga Alliance. [https://www. yogaalliance.org/SchooIPublicProfile?sid=1875\&lid=1875]. Accessed 17 Oct 2016.

46. Cope S. The wisdom of yoga. New York: Bantam Books; 2006

47. Carson JW, Carson KM, Gil KM, Baucom DH. Mindfulness-based relationship enhancement. Behav Ther. 2004;35:471-94.

48. Krishnamurti J. Choiceless awareness. Ojai: Krishnamurti Publications of America; 1992. 
49. Nguyen TD, Attkisson CC, Stegner BL. Assessment of patient satisfaction: development and refinement of a service evaluation questionnaire. Eval Program Plann. 1983;6(3-4):299-313.

50. Atkinson TM, Mendoza TR, Sit L, Passik S, Scher HI, Cleeland C, Basch E. The Brief Pain Inventory and its "pain at its worst in the last 24 hours" item: clinical trial endpoint considerations. Pain Med (Malden, Mass). 2010;11(3):337-46.

51. Cleeland CS, Ryan KM. Pain assessment: global use of the Brief Pain Inventory. Ann Acad Med Singapore. 1994;23:129-38.

52. Mendoza TR, Wang XS, Cleeland CS, Morrissey M, Johnson BA, Wendt JK Huber SL. The rapid assessment of fatigue severity in cancer patients: use of the Brief Fatigue Inventory. Cancer. 1999;85(5):1186-96.

53. Buysse DJ, Reynolds 3rd CF, Monk TH, Berman SR, Kupfer DJ. The Pittsburgh Sleep Quality Index: a new instrument for psychiatric practice and research. Psychiatry Res. 1989;28(2):193-213.

54. Carpenter JS, Andrykowski MA. Psychometric evaluation of the Pittsburgh Sleep Quality Index. J Psychosom Res. 1998;45(1):5-13.

55. Herrmann C. International experiences with the Hospital Anxiety and Depression Scale-a review of validation data and clinical results. J Psychosom Res. 1997;42(1):17-41.

56. Zigmond AS, Snaith RP. The hospital anxiety and depression scale. Acta Psychiatr Scand. 1983;67(6):361-70.

57. Bohlmeijer E, ten Klooster PM, Fledderus M, Veehof M, Baer R. Psychometric properties of the five facet mindfulness questionnaire in depressed adults and development of a short form. Assessment. 2011;18(3):308-20.

58. Baer RA, Smith GT, Lykins E, Button D, Krietemeyer J, Sauer S, Walsh E, Duggan D, Williams JMG. Construct validity of the five facet mindfulness questionnaire in meditating and nonmeditating samples. Assessment. 2008; 15(3):329-42.

59. Society AT. ATS statement: guidelines for the six-minute walk test. Am J Respir Crit Care Med. 2002;166(1):111-7.

60. Jones LW, Eves ND, Mackey JR, Peddle CJ, Haykowsky M, Joy AA, Courneya KS, Tankel K, Spratlin J, Reiman T. Safety and feasibility of cardiopulmonary exercise testing in patients with advanced cancer. Lung Cancer. 2007;55(2):225-32.

61. Jones LW, Hornsby WE, Goetzinger A, Forbes LM, Sherrard EL, Quist M, Lane AT, West M, Eves ND, Gradison M, et al. Prognostic significance of functional capacity and exercise behavior in patients with metastatic non-small cell lung cancer. Lung Cancer. 2012;76(2):248-52

62. Cella DF, Perry SW. Reliability and concurrent validity of three visualanalogue mood scales. Psychol Rep. 1986:59(2 Pt 2):827-33.

63. Jensen MP, Keefe FJ, Lefebvre JC, Romano JM, Turner JA. One- and two-item measures of pain beliefs and coping strategies. Pain. 2003;104(3):453-69.

64. McCracken LM, Vowles KE, Eccleston C. Acceptance of chronic pain: component analysis and a revised assessment method. Pain. 2004;107(1-2):159-66.

65. Vowles KE, McCracken LM, McLeod C, Eccleston C. The Chronic Pain Acceptance Questionnaire: confirmatory factor analysis and identification of patient subgroups. Pain. 2008;140(2):284-91.

66. Steinhardt MA, Dishman RK. Reliability and validity of expected outcomes and barriers for habitual physical activity. J Occup Med. 1989:31(6):536-46.

67. Bryk AS, Raudenbush SW. Application of hierarchical linear models to assessing change. Psychol Bull. 1987;101:147-58.

68. Carson JW, Keefe FJ, Affleck G, Rumble ME, Caldwell DS, Beaupre PM, Kashikar-Zuck S, Sandstrom M, Weisberg JN. A comparison of conventional pain coping skills training and pain coping skills training with a maintenance training component: A daily diary analysis of short- and longterm treatment effects. Journal of Pain. 2006;in press.

69. Schwartz JE, Stone AA. Strategies for analyzing ecological momentary assessment data. Health Psychol. 1998:17:6-16.

\section{Submit your next manuscript to BioMed Central and we will help you at every step:}

- We accept pre-submission inquiries

- Our selector tool helps you to find the most relevant journal

- We provide round the clock customer support

- Convenient online submission

- Thorough peer review

- Inclusion in PubMed and all major indexing services

- Maximum visibility for your research

Submit your manuscript at www.biomedcentral.com/submit
Biomed Central 